\title{
Control freaks
}

Citation for published version (APA):

Sorger, B., Scharnowski, F., Linden, D. E. J., Hampson, M., \& Young, K. D. (2019). Control freaks:

Towards optimal selection of control conditions for fMRI neurofeedback studies. Neuroimage, 186, 256-

265. https://doi.org/10.1016/j.neuroimage.2018.11.004

Document status and date:

Published: 01/02/2019

DOI:

10.1016/j.neuroimage.2018.11.004

Document Version:

Publisher's PDF, also known as Version of record

\section{Document license:}

Taverne

\section{Please check the document version of this publication:}

- A submitted manuscript is the version of the article upon submission and before peer-review. There can be important differences between the submitted version and the official published version of record.

People interested in the research are advised to contact the author for the final version of the publication, or visit the DOI to the publisher's website.

- The final author version and the galley proof are versions of the publication after peer review.

- The final published version features the final layout of the paper including the volume, issue and page numbers.

Link to publication

\footnotetext{
General rights Owners
rights.

- You may freely distribute the URL identifying the publication in the public portal. please follow below link for the End User Agreement:

www.umlib.nl/taverne-license

Take down policy

If you believe that this document breaches copyright please contact us at:

repository@maastrichtuniversity.nl

providing details and we will investigate your claim.
}

Copyright and moral rights for the publications made accessible in the public portal are retained by the authors and/or other copyright owners and it is a condition of accessing publications that users recognise and abide by the legal requirements associated with these

- Users may download and print one copy of any publication from the public portal for the purpose of private study or research.

- You may not further distribute the material or use it for any profit-making activity or commercial gain

If the publication is distributed under the terms of Article $25 \mathrm{fa}$ of the Dutch Copyright Act, indicated by the "Taverne" license above, 


\title{
Control freaks: Towards optimal selection of control conditions for fMRI neurofeedback studies
}

\author{
Bettina Sorger ${ }^{\mathrm{a}}$, Frank Scharnowski ${ }^{\mathrm{b}, \mathrm{c}, \mathrm{d}}$, David E.J. Linden ${ }^{\mathrm{e}, \mathrm{f}}$, Michelle Hampson ${ }^{\mathrm{g}}$, \\ Kymberly D. Young ${ }^{\text {h, }}$

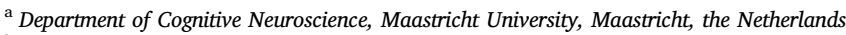 \\ ${ }^{\mathrm{b}}$ Department of Psychiatry, Psychotherapy and Psychosomatics, Psychiatric Hospital, University of Zürich, Zürich, Switzerland \\ ${ }^{\mathrm{c}}$ Neuroscience Center Zürich, University of Zürich and Swiss Federal Institute of Technology, Zürich, Switzerland \\ ${ }^{\mathrm{d}}$ Zürich Center for Integrative Human Physiology (ZIHP), University of Zürich, Zürich, Switzerland \\ e Cardiff University Brain Research Imaging Centre, School of Psychology, Cardiff University, Cardiff, United Kingdom \\ ${ }^{\mathrm{f}}$ School of Mental Health and Neuroscience, Faculty of Health, Medicine and Life Sciences, Maastricht University, Maastricht, the Netherlands \\ ${ }^{\mathrm{g}}$ Department of Radiology and Biomedical Imaging, Psychiatry and the Child Study Center, Yale School of Medicine, New Haven, CT, USA \\ ${ }^{\mathrm{h}}$ Department of Psychiatry, University of Pittsburgh School of Medicine, Pittsburgh, PA, USA
}

\section{A R T I C L E I N F O}

\section{Keywords:}

Neurofeedback

Real-time neuroimaging

Control conditions

Sham

Mental-rehearsal

Placebo

\begin{abstract}
A B S T R A C T
fMRI Neurofeedback research employs many different control conditions. Currently, there is no consensus as to which control condition is best, and the answer depends on what aspects of the neurofeedback-training design one is trying to control for. These aspects can range from determining whether participants can learn to control brain activity via neurofeedback to determining whether there are clinically significant effects of the neurofeedback intervention. Lack of consensus over criteria for control conditions has hampered the design and interpretation of studies employing neurofeedback protocols. This paper presents an overview of the most commonly employed control conditions currently used in neurofeedback studies and discusses their advantages and disadvantages. Control conditions covered include no control, treatment-as-usual, bidirectional-regulation control, feedback of an alternative brain signal, sham feedback, and mental-rehearsal control. We conclude that the selection of the control condition(s) should be determined by the specific research goal of the study and best procedures that effectively control for relevant confounding factors.
\end{abstract}

\section{Introduction}

Neurofeedback is increasingly coming into the focus of translational neuroscience research, both as a tool for understanding brain-behavior relationships and as a potential intervention for a variety of neurological and psychiatric diseases. A PubMed search of the term "neurofeedback" reveals that compared to 10 years ago the number of journal papers published on the topic has increased by $850 \%$. Neurofeedback can be employed using brain hemodynamics (as with functional magnetic resonance imaging [fMRI] or functional near-infrared spectroscopy [fNIRS]) as well as neuroelectric signals (as with electroencephalography [EEG], magnetoencephalography [MEG], or intracortical recordings). In this paper, we focus on fMRI neurofeedback, though many of our conclusions can be applied to other feedback methods, such as EEG. Neurofeedback contains several interacting features including the modification of brain activity during the procedure, the psychological processes involved in the self-regulation training, the instructions provided by the researcher, the high-tech environment, and the patients' expectancies associated with it. In a clinical context neurofeedback can thus be conceptualized as a complex intervention (Craig et al., 2008). Specific guidance is available for the development, outcome and process evaluation of complex interventions (Craig et al., 2008; Moore et al., 2015), which should be considered when designing neurofeedback trials. Careful documentation of the components of the intervention is an important part of the design, and the real time fMRI-neurofeedback community is working towards this documentation, as evidenced by a recent survey employing the Template for Intervention Description and Replication (TIDieR) checklist (Randell et al., 2018). Furthermore, neurofeedback researchers are encouraged to pre-register experiments and standardize measures, designs, statistical analysis, and reporting

\footnotetext{
* Corresponding author. Western Psychiatric Institute and Clinic, 3811 O'Hara Street, Pittsburgh, PA, 15213, USA.

E-mail address: youngk@pitt.edu (K.D. Young).
} 
(Thibault et al., 2018).

The stage of development of the intervention and the specific research focus - for example, assessment of feasibility, evaluation of efficacy or effectiveness - will determine whether randomized or non-randomized designs are more applicable, and in randomized designs, the choice of appropriate control conditions. Because neurofeedback is a complex intervention, there are numerous factors that must be considered and should be controlled for in order for causality to be unambiguously established. These factors include (Table 1):

- Participant Motivation/Perception of Success: Are participants actively trying to change their brain activity? Are differences in behavioral, clinical or brain changes across groups driven by differences in motivation/self-efficacy? This includes controlling for reward, frustration, and suspicion of control-group assignment.

- Neurophysiological Specificity: Is feedback from the target region required to obtain the desired changes in activity within that region or behavior to occur? Would simply gaining control (or perceiving to gain control) over brain activity more generally also result in these changes?

- Placebo/Expectation Effects/Participant Bias: What proportion of the behavioral/clinical effects is due to the use of a high-tech 'train-yourbrain' environment that participants believe to be effective?

- Non-Specific Effects: What proportion of the obtained activity/ behavior/clinical change is caused by more general factors? These factors may include arousal, perceptual input, and physiological parameters such as breathing and heart rate. Would simply changing breathing or arousal levels result in the same brain and behavioral/ clinical changes?

- Behavioral Effects: Would practicing a specific mental strategy to control brain activity in the absence of neurofeedback result in the same neural and behavioral/clinical changes? Is the neurofeedback component crucial in this context?

In this paper, we review the most commonly employed control conditions currently with a focus on which factors are (and are not) controlled for in each condition. These control conditions include (Fig. 1):

- No Control: No control condition is included; only within-subject changes are examined.

- No-Training Control: Participants do not receive any additional training. In clinical studies, this might include treatment-as-usual (TAU) or a waitlist control group.

- Bidirectional-Regulation Control: Participants are trained to selfregulate brain activity in opposite directions, or to self-regulate different aspects of brain function that induce the opposite behavioral changes.

- Placebo Control: Participants are provided with an alternative feedback signal.

○Neurofeedback of Alternative Brain Signal: Participants are provided with feedback of an alternative aspect of brain function. For example, in studies where the experimental group is trained to

Table 1

Explicit Control Conditions Commonly Employed in Neurofeedback Studies and the Confounding Factors they Address.

\begin{tabular}{|c|c|c|c|c|c|c|c|c|c|c|}
\hline \multirow{3}{*}{$\begin{array}{l}\text { Factors to be } \\
\text { controlled for to } \\
\text { establish causality }\end{array}$} & \multirow{3}{*}{ No control } & \multicolumn{2}{|c|}{ No-training Control } & \multirow{3}{*}{$\begin{array}{l}\text { Bidirectional- } \\
\text { Regulation } \\
\text { Control }\end{array}$} & \multicolumn{4}{|c|}{ Placebo Control } & \multicolumn{2}{|c|}{ Mental-Rehearsal Control } \\
\hline & & \multirow[t]{2}{*}{$\begin{array}{l}\text { Treatment } \\
\text { as usual }+\end{array}$} & \multirow{2}{*}{$\begin{array}{c}\text { List of } \\
\text { matched } \\
\text { participants/ } \\
\text { Waitlist+ }\end{array}$} & & \multicolumn{2}{|c|}{ Alternative feedback } & \multicolumn{2}{|c|}{ Sham feedback } & \multirow{2}{*}{$\begin{array}{l}\text { Inside the MRI } \\
\text { scanner }\end{array}$} & \multirow{2}{*}{$\begin{array}{l}\text { Outside the MRI } \\
\text { scanner }\end{array}$} \\
\hline & & & & & $\begin{array}{c}\text { Feedback from an } \\
\text { Alternative Brain } \\
\text { Signal }\end{array}$ & $\begin{array}{c}\text { Feedback } \\
\text { based on non- } \\
\text { brain signals }\end{array}$ & $\begin{array}{c}\text { 'Yoked' } \\
\text { feedback }\end{array}$ & $\begin{array}{l}\text { Artificially } \\
\text { generated } \\
\text { feedback }\end{array}$ & & \\
\hline $\begin{array}{l}\text { Equal Motivation/ } \\
\text { Perception of } \\
\text { Success }\end{array}$ & & & & & & & & & & \\
\hline $\begin{array}{c}\text { Demonstrate } \\
\text { neurophysiological } \\
\text { specificity }\end{array}$ & & & & & & & & & & \\
\hline $\begin{array}{c}\text { Exclude } \\
\text { placebo effects }\end{array}$ & & & & & & & & & & \\
\hline $\begin{array}{c}\text { Exclude } \\
\text { global (spatially } \\
\text { non-specific) } \\
\text { effects }\end{array}$ & & & & & & & & & & \\
\hline $\begin{array}{c}\text { Exclude } \\
\text { Behavioral effects }\end{array}$ & & & & & & & & & & \\
\hline Remarks & $\begin{array}{l}\text { Extremely } \\
\text { economical }\end{array}$ & $\begin{array}{l}\text { Economical } \\
\text { Crucial (and } \\
\text { fairest) } \\
\text { control } \\
\text { group in } \\
\text { clinical } \\
\text { context }\end{array}$ & $\begin{array}{c}\text { Very } \\
\text { economical }\end{array}$ & $\begin{array}{l}\text { Up-and } \\
\text { downregulati } \\
\text { on of the } \\
\text { same region } \\
\text { might not } \\
\text { always be } \\
\text { possible } \\
\text { Not always } \\
\text { ethically } \\
\text { justifiable+ }\end{array}$ & $\begin{array}{l}\text { Regions' signal } \\
\text { properties } \\
\text { should be } \\
\text { matched across } \\
\text { groups } \\
\text { Might be too } \\
\text { conservative } \\
\text { (critical region } \\
\text { might be trained } \\
\text { via functional } \\
\text { connectivity) } \\
\text { Risk of } \\
\text { unblinding } \\
\text { participants }\end{array}$ & $\begin{array}{c}\text { Risk of } \\
\text { unblinding } \\
\text { participants }\end{array}$ & $\begin{array}{c}\text { Risk of } \\
\text { unblinding } \\
\text { participants }\end{array}$ & $\begin{array}{c}\text { Should be } \\
\text { generated } \\
\text { considering } \\
\text { properties of } \\
\text { the } \\
\text { hemodynami } \\
\text { c response } \\
\text { Risk of } \\
\text { unblinding } \\
\text { participants }\end{array}$ & $\begin{array}{l}\text { Not always } \\
\text { possible } \\
\text { (implicit } \\
\text { neurofeedback) } \\
\text { Control } \\
\text { participants } \\
\text { should not be } \\
\text { aware of the } \\
\text { existence of the } \\
\text { neurofeedback } \\
\text { group }\end{array}$ & $\begin{array}{c}\text { Not always } \\
\text { possible } \\
\text { (implicit } \\
\text { neurofeedback) } \\
\text { Can } \\
\text { considerably } \\
\text { minimize } \\
\text { scanning costs } \\
\text { Control } \\
\text { participants } \\
\text { should not be } \\
\text { aware of the } \\
\text { existence of the } \\
\text { neurofeedback } \\
\text { group }\end{array}$ \\
\hline
\end{tabular}

\footnotetext{
* if ability to control is balanced across groups

$\wedge$ if subjects are not unblinded by signal not matching mental changes

@ if control signal does not have an antagonistic relationship with the behavioral/clinical variable of interest.

+ signifies a situation in a clinical context.
} 


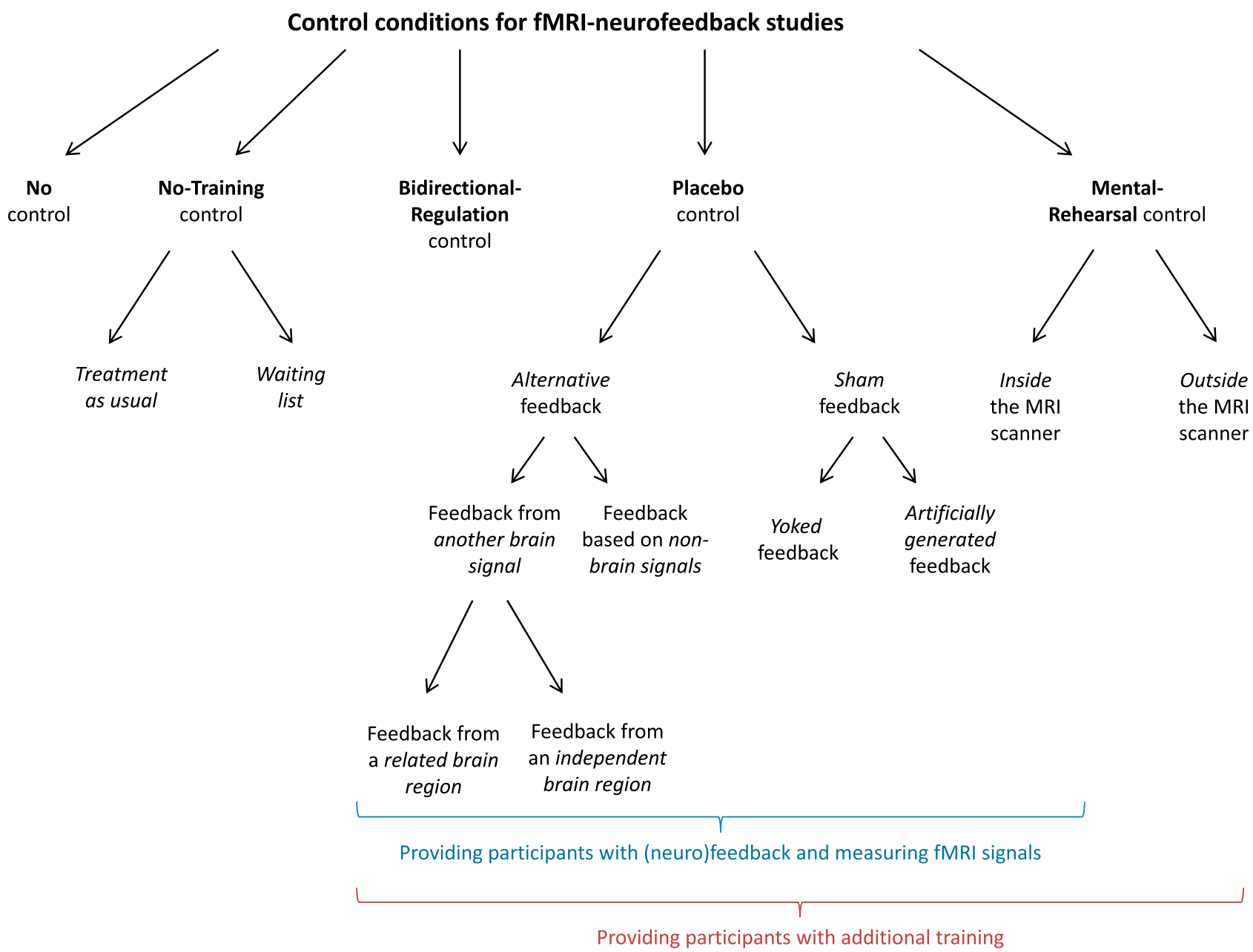

Fig. 1. Overview of control conditions for neurofeedback studies.

control activity in a specific brain region, the control group may be trained to modulate activity in a different brain region. oNon-Neural Feedback: Participants are provided with feedback based on non-brain signals. These signals can include various physiological measures such as breathing, heart rate, or skin conductance.

oSham Feedback: Participants are not provided with their own feedback signal. Instead a signal of another participant ('yoked feedback') or an artificially generated signal is presented.

- Mental-Rehearsal Control: In the absence of any feedback information, participants engage in the same mental strategy that is used by participants in the experimental group, or a strategy that is known to be related to the trained brain signal.

\section{When is No control acceptable?}

Early phases of development and evaluation of a novel medical intervention may occur without direct clinical testing or implementation of control conditions. Both the US (https://wwwnimhnihgov/about/ directors/thomas-insel/blog/2012/experimental-medicineshtml2012) and the UK medical funding organizations (https://www.mrc.ac.uk/ documents/pdf/rcts-for-complex-interventions-to-improve-health/)

have provided guidelines for phased development and evaluation of complex interventions. In the case of neurofeedback, the "preclinical" or "theoretical" phase may entail the identification of target brain signals or states based on meta-analyses of published functional neuroimaging studies, the programming/development of the appropriate experimental protocols/brain-computer interfaces (BCIs) and the testing of the setup in healthy individuals. The "modelling" phase can involve experimental brain-imaging studies and comparison of different neurofeedback protocols in healthy participants or patients, but also exclusively rely on modelled data obtained from simulations. It has recently been shown that simulation studies can be useful for the assessment of feasibility of realtime fMRI neurofeedback protocols (Oblak et al., 2017). The next stage would be an "exploratory trial", which could employ an adaptive design, in order to optimize the intervention and assess its feasibility in the targeted patient population. Parameters of feasibility would include the ability of patients to attain the desired level of control of the targeted measure of brain activity (e.g., up- or downregulation of the mean hemodynamic signal across a brain region or network, up- or downregulation of a parameter of functional connectivity, or increasing similarity of a brain-activity pattern to a template identified by machine learning), patient adherence more generally (e.g., assessed by debriefing), determination of dosing (number of sessions required for a change in brain and/or behavior to occur), patient retention, and patient satisfaction. The fidelity of treatment delivery by the research team or therapists is also an important consideration when designing reliable intervention protocols (Persch and Page, 2013). This approach is similar to Phase I Clinical Trial designs which aim only to determine the safety and feasibility of the novel treatment/procedure (Spilker, 1991). No control group is required to address these questions, and a single-group design would thus normally be appropriate at this stage.

In single-group designs, while repeated measures are used, nonspecific effects (including placebo, motivation, and simple practice effects) cannot be ruled out. For studies examining changes in symptoms over time, particularly those examining psychiatric symptoms, regression 
towards the mean and natural recovery are particularly problematic without an appropriate control (Paulus et al., 2014). In other (particularly chronic and progressive) disorders, such as Parkinson's disease where spontaneous remission is not observed, within-subject changes can indeed allow for some conclusions to be drawn regarding the efficacy of the intervention, but placebo effects cannot be excluded.

Many fMRI-neurofeedback studies that have not included control conditions have been labeled as "pilot," "feasibility," or "preliminary" studies in the title (Buyukturkoglu et al., 2015; Sitaram et al., 2014; Li et al., 2013; Gerin et al., 2016). Studies lacking a control condition can be cost-effective for making decisions about whether to continue with the particular line of research. It is particularly important in single-group designs to examine differences in outcome/behavior between participants that are successful at learning to regulate the signal and those who are unsuccessful. Half of fMRI neurofeedback studies fail to find participants able to regulate the target hemodynamic signal or find mixed results regarding regulation success and (Thibault et al., 2018), and it is estimated that approximately $30 \%$ of participants will not be able to learn to control a signal across neurofeedback designs (Allison and Beuper, 2010). Examination of these subgroups can assist in the development of subsequent randomized trials that minimize the number of non-learners to avoid loss of power and waste of resources by making the neurofeedback task more learnable and/or identifying predictors for patient selection. Demonstrating that regulators improve on the outcome measure relative to the unsuccessful regulators would allow for some conclusions regarding brain-behavior relationships to be established, though it is possible that the non-learners are simply unmotivated and are not actively engaging in the regulation strategy. However, causality cannot be established without appropriate control conditions. Such non-randomized studies may provide useful information about outcomes, for example, effect sizes of clinical improvements that can inform the sample calculations for definitive clinical trials. It is critical, however, that authors clearly acknowledge the limitations of having no control condition in their publication.

\section{Commonly employed control conditions}

Once feasibility has been established, experimental designs that focus on outcome evaluation are warranted. While fMRI neurofeedback may not currently be a very cost-effective intervention, carefully designed studies using this methodology can lead to a better understanding of the mechanisms underlying onset and recovery from particular disorders and ultimately lead to more cost-effective and directed treatments. Once a randomized clinical trial (RCT) is justified, the question of control intervention gains prominence. Compared to drug trials, for which the gold standard is the "placebo", the options and consequently the challenges in the design of complex intervention trials are more numerous. Most "placebo" interventions will also entail an active component.

\subsection{No-Training Control}

At some point in intervention development, the comparison of neurofeedback, either as a stand-alone or add-on intervention, to TAU is desirable (Cox et al., 2016). This design may be particularly useful in scenarios where TAU is widely available and can reveal whether the neurofeedback component is likely to have a clinically significant benefit over available treatments. Whether neurofeedback has benefits over available treatments is in many respects the core clinical question. As fMRI neurofeedback is relatively expensive and at this time not widely available, the question of whether an fMRI neurofeedback intervention is effective, or an effective addition, to cheaper and more widely available treatments becomes crucial. The answer can inform decisions whether to invest in further development and evaluation of the neurofeedback intervention for a particular indication, or indeed whether, assuming the safety profile is favorable (which is currently deemed to be the case for neurofeedback (Hawkinson et al., 2012)), to make it readily available as a clinical service. Of course, the neurofeedback plus TAU vs. TAU only comparison does not address any non-specific effects. These effects would have to be addressed by comparison with placebo control interventions. Furthermore, this comparison does not answer the question whether other interventions, for example those using cheaper biofeedback technology, would have similar effects. This comparison may also be too conservative in settings in which TAU is already very effective and no major added benefit is expected from a complex intervention, which may, however, still be effective if delivered on its own. Direct comparison with TAU would be required to address this question. However, direct comparisons with TAU would realistically only be done for conditions in which TAU is either expected to be more expensive than neurofeedback (which may be the case for resource-intensive psychotherapies or for expensive proprietary drugs) or entails major side effects or compliance problems.

It has been argued that because there is no true "consistent background" practice against which any new intervention can be tested, TAU is an inadequate control and should be "removed from our scientific vocabulary" (Burns, 2009). Therefore, to allow for replication and interpretation of results, it is critical that precise details about this treatment be provided. This includes information about who provided the treatment, what the treatment consisted of, number and duration of sessions, and the country and standard of health care provided (Watts et al., 2015).

Comparison to a wait-list control group may exaggerate the apparent efficacy of the intervention. For example, participants in the control group may be motivated to stay depressed so they are still eligible when the wait period is over (Furukawa et al., 2014).

\subsection{Bidirectional-regulation control}

Many real-time fMRI neurofeedback studies train participants to either increase or decrease the mean activity level within a particular brain region or network in order to achieve a specific behavioral/clinical effect. Training the same participants or another group of participants to regulate this activity in the opposing direction should cause opposing behavioral effects, thus allowing for a powerful control condition.

In neurofeedback methods development, voluntary bidirectional control of a new feedback signal has been used to demonstrate feasibility of that signal for training (Koush et al., 2013; Sacchet et al., 2012). For example, before using a new connectivity signal for real-time fMRI neurofeedback training, investigators tested if each participant could upand down-regulate the connectivity signal through cognitive task performance (Koush et al., 2013). Whereas unidirectional control can easily be achieved through non-specific effects such as arousal, bidirectional control is much less prone to artefactual self-regulation (though it is possible that participants could alter breathing, muscle, or arousal levels both up and down to achieve bidirectional control).

Another advantage of using bidirectional control is that it allows for ruling out motivational and placebo effects. Motivational differences might exist between baseline and regulation conditions, but not between regulating in different directions (provided that regulating the signal in both directions is equally feasible). Note however, that even if motivation differs between the different training directions (possibly because controlling in one direction is easier than the other), conclusions can still be drawn regarding brain-behavior relationships if the changes in behavioral measures from baseline in the two conditions are in opposite directions. Likewise, placebo effects might cause behavioral improvements (or worsening), but they cannot explain opposing behavioral effects as a result of bidirectional control, provided that participants are equally successful at regulating each direction. However, participant-bias still needs to be excluded, otherwise knowledge about the function of the targeted brain region (for example being trained to up- and downregulating anxiety-related brain areas) might yield corresponding in-/ decreases in anxiety. This effect could be controlled for using implicit training protocols where participants are unaware they are engaging in a 
neurofeedback task, and in some instances do not know they are being trained at all (see Section 4 for more detail) and careful blinding of conditions so that participants do not know what condition they are in, and expectations are controlled for.

Several variants of bidirectional control exist, using either a within- or a between-group design, training bidirectional control of the same or of different aspects of brain function, and even combining bidirectional control with differential feedback. For example, in a between-group design, Shibata et al. trained one group to up-regulate a brain activity pattern associated with high facial preference, and another group to upregulate an activity pattern associated with low facial preference (Shibata et al., 2016). They found that faces previously rated as neutral became more (high facial preference group) or less (low facial preference group) preferred. Here, bidirectional control does not refer to up- or down-regulating the same region of interest (ROI), but to training physiologically specific brain patterns to induce opposing behavior. Alternatively, Scharnowski et al. (2015) used a within-group design to simultaneously up- and down-regulate two functionally distinct ROIs (the supplementary motor area (SMA) and the parahippocampal cortex (PHC)) using differential feedback (i.e., SMA minus PHC activity). After confirming that participants were able to up- and down-regulate both regions, they tested the effects of such regulation on behavioral tasks related to the function of the respective ROIs, i.e., a motor reaction time task related to SMA activity and a memory task related to PHC activity. This combination of up- and down-regulating functionally unrelated ROIs with ROI-specific behavioral testing allows for a strong double dissociation to derive specific inferences about localized brain function (Teuber, 1955).

Overall, bidirectional control is a powerful control condition that can be used to ensure physiological specificity, can control for placebo and motivational effects (provided that participant biases can be controlled for), and rules out non-specific effects (Table 1). This control condition can be used for between-as well as within-group designs, and it has been successfully used in biofeedback, magnetoencephalography (MEG)-, EEG-, and fMRI-neurofeedback studies (Sacchet et al., 2012; Shibata et al., 2016; Scharnowski et al., 2015; Colgan, 1977; Brody et al., 1994; Blankstein et al., 1976; Cortese et al., 2016, 2017; Hinterberger et al., 2003; Potolicchio et al., 1979; Regestein et al., 1973). However, bidirectional control does not exclude that mental rehearsal alone would be sufficient to change brain activity and associated behavior. Another limitation is that bidirectional control is not always desirable, especially in clinical contexts where training the opposite direction might cause unwanted behavioral consequences (i.e., worsening of symptoms) in patients. This, however, is predominantly a precautionary measure because there is little evidence suggesting negative outcomes from fMRI neurofeedback training regardless of the regulation direction. Finally, alternating up- and down-regulation in a within-group design can induce order and carry-over effects that can affect the behavioral outcome and might make learning more difficult (Cortese et al., 2016, 2017).

\subsection{Placebo control}

\subsubsection{Feedback of an alternative brain signal}

In this type of control condition, participants receive veritable neurofeedback, but of an aspect of brain function other than that targeted in the experimental group. Optimally, participants should be able to gain a similar level of control over the trained signal as the experimental group and any assigned mental strategy used should be the same across groups. This approach controls for psychoeducative effects (the benefit of learning to control a signal) and allows for conclusions as to whether feedback of the target aspect of brain function is necessary to gain control over that aspect. Additionally, this control condition allows for examination of whether changes in mood and behavior in the experimental group are due specifically to the feedback or to a placebo effect. As long as participants are equally successful at regulating the neurofeedback signal, motivation effects should also be controlled for (Table 1). For simplicity in our discussion here, we will assume a single brain region is trained in the experimental group, and another brain region is trained in the control group, although the concepts can be generalized to more complex aspects of brain function that are trained, such as distributed activity patterns, or functional connectivity patterns.

When designing this type of control condition, it is important that the control ROI is matched to the target ROI in terms of either tissue composition, number of voxels, and/or temporal signal-to-noise ratio so that accurate and reliable detection of activation is possible and different results are not due to one region being easier to regulate because of better signal properties (Murphy et al., 2007; Young et al., 2017; Paret et al., 2014). Furthermore, the control ROI should be functionally independent from the target ROI (Young et al., 2017; Paret et al., 2014). Studies using this design can be challenging to develop as it can be difficult to select a control ROI that is both independent from the target ROI (so that regulating the control ROI does not systematically affect the target ROI's activity), and that can be regulated by participants as easily as the target ROI using the same assigned mental strategy (if a mental strategy is assigned) (Young et al., 2017; Garrison et al., 2013; Alino et al., 2016). If participants are unable to regulate the control ROI, they may become frustrated and give up trying to regulate the signal. Then, any difference between the experimental and control groups is not just due to the region regulated, but whether any regulation could be achieved at all. Also, if participants are unable to regulate the control ROI as well as the experimental group, even if they are equally motivated, they will receive less rewarding feedback and consequently their placebo effects may be smaller. Neurofeedback research using this control methodology has produced mixed results: In an fMRI-neurofeedback training study with children with attention deficit hyperactivity disorder (ADHD), gaining control over any signal (the target or control ROI) resulted in clinical improvement, suggesting it may be the feeling of self-efficacy or learned control over hemodynamic activity in general that leads to clinical improvements in individuals with this disorder (Alegria et al., 2017). In adults with major depressive disorder, however, learning to regulate a parietal region did not result in symptom improvement, while learning to regulate the amygdala did (Young et al., 2017). Several studies employing this control condition did not find that participants were able to learn to regulate the control ROI (Paret et al., 2014; Guan et al., 2015; Lawrence et al., 2014). In these studies, the effects of success, feelings of self-efficacy, and frustration cannot be ruled out as explaining group differences.

When participants are equally successful at regulating the assigned ROI, this control condition can allow for strong conclusions, specifically that (a) information from the ROI is needed to gain control over that ROI and for behavioral/clinical changes to occur, and (b) gaining control over any signal more generally would not result in the same effects. While behavioral effects cannot be completely controlled for (it is still possible that engaging in the mental strategy only in the absence of feedback information would result in the same clinical and neurophysiological changes), behavioral effects are controlled for to some extent when both groups are instructed to use the same strategy to control a brain region. In this case, group differences in outcome measures are not due to engaging in the strategy while learning to control a signal. However, the difficulty and intense pilot testing necessary to ensure the selection of an appropriate control ROI make it particularly difficult to successfully implement this condition.

\subsubsection{Non-Neural Feedback}

Neurofeedback is a special type of biofeedback. In biofeedback, a physiological measure is obtained and fed back to participants. In the case of neurofeedback the physiological measure is the hemodynamic response (measured with fMRI or fNIRS) or electrical activity (measured with EEG or MEG). Comparison of the effects of neurofeedback to other biofeedback methods is another type of control. As heart-rate variability and respiration biofeedback have been shown to reduce symptoms in patients with anxiety and depressive disorders (Schoenberg and David, 
2014), using this control condition has the advantage of comparing neurofeedback to a similar effective intervention. If subjects are equally successful at learning to regulate the control signal as the experimental signal, this approach can control well for motivation/perceived success, as well as non-specific factors (Table 1). This comparison may or may not control for placebo effects related to receiving a high-tech, cutting-edge intervention, depending on whether the subject's perception of the experimental and control interventions is comparable in this respect.

Non-neural feedback has been widely used in EEG-neurofeedback studies; but to date only one fMRI neurofeedback study has included it (deCharms et al., 2005). In this study, autonomic tone feedback (including measures of skin conductance, heart rate, and respiration) associated with decreased arousal and increased relaxation was significantly less effective at reducing perception of pain than neurofeedback from the anterior cingulate cortex (deCharms et al., 2005). Studies have found EEG neurofeedback to be superior to respiration feedback in reducing seizures in patients with epilepsy (Kotchoubey et al., 2001), to electromyography (EMG) feedback in reducing ADHD scores in children (Baumeister et al., 2016), and to skin-conductance feedback training in improving cognitive flexibility in autism (Kouijzer et al., 2013). Other studies, however, have found EEG neurofeedback to be as effective as EMG feedback in reducing ADHD symptoms in children (Maurizio et al., 2014), and heart rate-variability training to reduce anxiety more than EEG-neurofeedback training in dance students (Gruzelier et al., 2014).

\subsubsection{Sham feedback}

In a sham control condition, participants are provided with feedback information that is not based on their actual brain signal. This signal can be artificially (randomly or systematically) generated feedback which involves creating a signal that is not based on an actual brain imaging signal. Typically, the signal shares crucial properties with the real neurofeedback signal, including similar frequency, amplitude, and in the case of fMRI neurofeedback training, taking into account the hemodynamic delay (Johnson et al., 2012; Mihara et al., 2012).

Sham feedback can also involve yoked feedback, where each control participant sees the actual feedback signal from a participant in the experimental group. The sham condition is one of the most commonly employed control conditions in neurofeedback studies (Alino et al., 2016). The benefits of this control condition include matching the experimental condition on all aspects except gaining control over the experimental ROI signal. Provided that control participants do not detect the non-contingency between their efforts and the resulting signal change, there should be equal motivation and perceived success between the groups (Table 1). However, this control condition cannot determine if the mental strategy alone would result in similar behavioral and brain changes, although the same mental strategies are typically recommended to subjects in both groups to reduce this concern. However, when participants do detect non-contingency, negative effects, such as frustration and decreased motivation can become critical confounds. While some studies have reported that participants in yoked neurofeedback conditions did indeed notice the non-contingency (Johnson et al., 2012; Sulzer et al., 2013a; Kravitz et al., 2996), many studies have reported that participants remained unaware of this non-contingency (Chiew et al., 2012; Ninaus et al., 2013; Witte et al., 2013; Hamilton et al., 2016; deCharms et al., 2004). Unfortunately, many studies employing yoked feedback do not report on whether the blind was maintained or monitor frustration effects (Johnson et al., 2012; Hamilton et al., 2011; Caria et al., 2007; Lee et al., 2012; McCaig et al., 2011; Subramanian et al., 2011; Rota et al., 2009), leaving open the possibility that the differences between the yoked and experimental groups were due to motivation effects and not to gaining control over the signal. Non-contingency is less likely to be detected in feedback studies that provide intermittent or delayed feedback where a summary is given after the regulation period as opposed to real-time feedback studies where the signal is updated continuously (e.g., every two seconds) (Johnson et al., 2012). When employing sham feedback, monitoring frustration/satisfaction during the study, and asking subjects after the study if they believe they had received the experimental intervention (and having them rate how confident they are in that belief) are critical for interpretation of results.

The perception that the feedback signal is not contingent with effort will not necessarily differ between groups in a sham controlled study. Many participants who receive real neurofeedback signals do not feel that the signal changes reflect their effort (because of the high difficulty of controlling the signal). Thus, non-contingency with effort (and resulting frustration) is a common element of neurofeedback studies regardless of group assignment. Assuming participants are trying consistently, contingency of effort and signal change are likely to be similar in magnitude in the sham group and experimental neurofeedback groups (when the increase/decrease blocks are time-locked) because of the balancing of the perception of success. However, patients who are aware that sham feedback is a control condition in the study may be more prone to suspect non-contingency regardless of which group they were assigned to. This awareness may reduce overall clinical improvements that can be achieved in sham controlled designs.

\subsection{Mental-rehearsal control}

It is important to note that for all of the control conditions discussed thus far in which subjects are instructed to use a specific mental strategy during neurofeedback, there is invariably one factor that cannot be controlled for - and that is whether strategy alone would cause the same neural or behavioral changes. We refer to this here as a mental-rehearsal, no feedback, or strategy only control. Here, control subjects are instructed to self-regulate brain activity in the absence of any feedback information by repeatedly applying a specific self-regulation strategy that was communicated to them before the start of the training. The mental strategies provided to the control subjects should be identical to those provided to participants receiving neurofeedback information prior to the start of training. These strategies should be guided by theoretical considerations and empirical evidence from literature, as well as on the experience of study participants who have undergone the neurofeedback task. The selected mental strategy must be clear and unambiguous and should be provided in written form to participants. It is also advisable to verify participants that understand the mental strategy to be used.

Within a sample of 99 studies systematically analyzed in a recent literature review on fMRI-neurofeedback experiments (Thibault et al., 2018), 17 studies employed this control condition. This control condition has been often criticized due to its differences in terms of expectation and motivation when compared to the neurofeedback situation. However, the mental-rehearsal control holds several crucial advantages and can play a critical role in demonstrating a true neurofeedback effect because it provides the only possibility to control for the effects of simply engaging in the behavioral/cognitive strategy without the help of any additional (e.g., neurofeedback) information. Additionally, in specific situations, mental rehearsal constitutes the only meaningful control condition (e.g. in communication and control brain-computer interface (BCI) studies that involve a neurofeedback approach or when investigating the neural correlates and mechanisms of fMRI-neurofeedback (Sorger et al., 2016; Krause et al., 2017)). For example, Sorger and colleagues (Sorger et al., 2016) investigated whether varying the loudness of inner speech (a mental-strategy control) aids in gradual brain-activity regulation. The researchers tested both whether the gradual modulation ability was pre-given (no-feedback condition/mental task performance only) or could be further enhanced by providing participants with continuous feedback about the current brain-activity level in a mental-task-specific brain region (feedback condition). To include a sham-feedback condition in this instance would not have been a meaningful option as sham feedback is not expected to further increase the gradual modulation ability. Of course, it might still be possible that sham neurofeedback could motivate participants to try harder and in turn help them alter the behavior in question in a gradual manner.

Generally, the mental-rehearsal control condition can be easily 
applied, as its implementation is technically less challenging than the implementation of any placebo control condition. Moreover, this control condition does not suffer from the ethical limitations that might emerge in placebo feedback control conditions (see discussion in section 3.5). While the mental-rehearsal control condition can be used both in withinand between-group designs, the latter should be preferred to prevent undesirable order and carry-over effects (see, e.g., (Sorger et al., 2016)). Mental-rehearsal can be employed as control condition for both continuous- and intermittent-neurofeedback designs. Compared with intermittent neurofeedback, mental rehearsal is considerably easier to match to the neurofeedback condition in terms of sensory input and the levels of cognitive demand/work load.

The mental-rehearsal control condition can be implemented both inside (Caria et al., 2007, 2010; McCaig et al., 2011; Subramanian et al., 2011; Sorger et al., 2016; Krause et al., 2017; Bray et al., 2007; Greer et al., 2014; Habes et al., 2016; Harmelech et al., 2015; Hartwell et al., 2016; Johnston et al., 2011; Li et al., 2016; MacInnes et al., 2016; Marins et al., 2015; Megumi et al., 2015; Moll et al., 2014; Sarkheil et al., 2015; Yoo et al., 2006; Zhang et al., 2013; Zilverstand et al., 2015, 2017) and outside the MRI scanner (e.g., (deCharms et al., 2005; Linden et al., 2012; Sherwood et al., 2016a; Sherwood et al., 2016b; deBettencourt et al., 2015)). The latter option is much easier to realize and considerably decreases associated (personnel and scanning) costs. For this reason, mental rehearsal might provide a more economical solution in case of limited resources. However, implementing this control condition inside the MRI scanner has several advantages: global, spatially non-specific (e.g., general-arousal) effects can be ruled out later by analyzing the functional brain-imaging data that is obtained during mental rehearsal; and placebo and motivational effects can be partially controlled for because participants are placed in the same high-tech MRI environment (though not experiencing the neurofeedback setup). Another possibility to control/reduce motivational effects related to the MRI environment itself might be to implement the mental-rehearsal control condition in a dummy (or 'mock') scanner. It might be challenging to make participants believe that they are in a true MRI scanning environment but it would allow matching at least a few additional factors. Global motivational effects can be further limited when control subjects are not informed about the existence of the neurofeedback group, as awareness that one does not belong to the main experimental group might considerably lower a participant's motivation. Instead, participants might be told that they are participating in a training study applying a specific mental strategy in which brain activity will be measured at the same time in order to trace the training effect in the brain (see discussion in Zilverstand et al., 2015, 2017). Frustration is a potential confound even when receiving veritable feedback, in case of failure to control the neurofeedback signal. Participants in the mental-rehearsal control condition, however, are less likely to be frustrated as they do not expect nor receive any information on their current performance. On the other hand, they may have reduced motivation compared to the experimental group because the feedback is generally engaging and they are not receiving any.

Despite many features, using the mental-rehearsal control condition alone is not sufficient to exclude all possible alternative explanations for an obtained positive behavioral neurofeedback effect. Most importantly, mental rehearsal cannot rule out motivational and placebo effects as this control condition does not include the feedback component. While this control condition is helpful in establishing a true neurofeedback effect by controlling for pure behavioral effects, mental rehearsal should ideally be combined with one or more other control conditions (see Table 1), either in the same or consecutive studies.

\subsection{Practical implementation}

\subsubsection{What to disclose to participants}

One issue that is particularly important in the context of control conditions is the degree of disclosure to participants regarding the study design. If participants are told that there is a sham feedback group, it can make them more likely to look for a mismatch between the feedback signal and what they expect the brain region to be doing, and thus can make them more likely to guess that they are not getting veritable neurofeedback. This issue must also be considered with alternative ROI controls. For example, if the goal is to treat anxiety symptoms and participants are told that they will either be trained on an anxiety-related brain region or a region unrelated to anxiety, control participants may be more likely to notice if the feedback does not match their anxiety and to suspect they are getting the control intervention. Having figured out their group allocation, it is possible that these participants will assume that their symptoms could not have improved and this assumption may influence the behavioral effects. In some clinical trials, vagueness is used and participants are simply told that there are two conditions they can be assigned to randomly (an experimental intervention that may improve symptoms and a control intervention that is not expected to improve symptoms) but not about the precise difference between these two conditions. Non-authorized deception, where participants are not told of the existence of a control condition until after completion of the study (as opposed to authorized deception where researchers inform participants beforehand that deception will be used, but not how), may be an acceptable option, provided that participants are then offered the experimental intervention following completion of the study protocol. How much researchers are required to disclose to participants varies across different institutions, depending on the particular legal environment and requirements from the institutional review board (IRB), and may affect what the best control option is for a given group.

\subsubsection{Ethical concerns}

Research using sham or alternative ROI feedback has raised ethical concerns for researchers (Alino et al., 2016; Thibault et al., 2016; Ruiz et al., 2013; Kluetsch et al., 2014). One concern is that researchers are withholding effective treatment, violating the conditions set forth in the declaration of Helsinki (Carlson et al., 2004). Neurofeedback, however, has yet to achieve the status of "evidence-based medicine" and therefore controlled studies are not withholding established effective treatment (Sulzer et al., 2013b). Indeed, the goal of many of these studies is to determine whether neurofeedback might be developed into an effective treatment. Furthermore, researchers could administer experimental neurofeedback to all participants after completion of the experiment to ensure that all participants eventually received the investigational treatment (Carlson et al., 2004), as is common procedure in pharmacological clinical trials.

\section{Special case: implicit neurofeedback}

The choice of a control condition not only depends on what aspect one wants to control for, but also on the experimental neurofeedback approach itself. To date, most fMRI neurofeedback training studies have followed an explicit approach where participants are informed about the neurofeedback target region(s) and potential regulation strategies. In contrast, implicit neurofeedback is characterized by training participants without providing them with any information regarding the trained brain region, what the feedback signal represents, or potential mental strategies (Watanabe et al., 2017). In fMRI neurofeedback, this approach is often characterized by training participants to match ongoing brain activity to a multivariate brain activity pattern that is associated with a specific behavior, mental, or perceptual state (coined "Decoded Neurofeedback" or 'DecNef' (Shibata et al., 2011)), but may also be applied when following the more classical neurofeedback approach of changing the mean activity level within a particular brain region or connectivity measure of a network. It is difficult to run a mental-rehearsal control group with this paradigm, because the experimental group is not provided with strategy suggestions, and debriefing of participants that have been trained with implicit neurofeedback indicate diverse mental activities that often seem unrelated to the brain activity pattern that was 
trained (Shibata et al., 2016).

Instead of including a mental-rehearsal control group, in certain implicit neurofeedback paradigms, one can make sure that behavioral effects are primarily due to neurofeedback by training only one specific brain state, e.g., a visual-cortex activity pattern corresponding to a $70^{\circ}$ grating orientation, and testing visual sensitivity for gratings of different orientations. Using this approach, Shibata et al. found improved visual sensitivity only for the trained grating orientation but not for other grating orientations, thus ensuring physiological specificity of the behavioral effect (Shibata et al., 2011). In this way, placebo, non-specific and motivation effects can also be excluded, because these effects are not specific to only the target grating orientation which is unknown to the participants. Another example comes from Koizumi et al., in which participants were reinforced when their brain activity reflected a pattern in the visual cortex associated with a fearful stimulus in the absence of stimulus presentation (Koizumi et al., 2016). This training reduced the fear response (measured by skin conductance response) to the trained conditioned stimulus (i.e., green circle) but not to untrained but conditioned stimuli (i.e., red circle). In this example, there is no control condition per se, but instead there is a control outcome measure - ensuring the specificity of the effects by demonstrating that only the targeted behavior has changed.

\section{Discussion}

There are numerous options for control conditions in neurofeedback studies, and there is no single best control condition that addresses all potential confounding factors. This conclusion may lead researchers to believe that they need to include all of the control conditions discussed into a single study. However, this approach would be very elaborate and costly and therefore not realistic considering limited resources and the negative effects of multiple-group designs on power. The most comprehensive study to date employed five different control conditions including behavioral training only (mental rehearsal), twice the amount of behavioral training as neurofeedback training, alternative ROI feedback, yoked feedback, and autonomic biofeedback (deCharms et al., 2005). Only participants in the experimental group receiving anterior cingulate neurofeedback reported decreased perception of pain, leading the authors to conclude that it is gaining control of a particular regional signal that leads to the observed changes in pain perception, and not effects of motivation, expectation, or non-specific effects. Unfortunately, the sample was very small (only 4-8 participants in each group) and the authors were unable to replicate their results (Sulzer et al., 2013b). Thus, ensuring properly powered studies is also an important consideration when designing neurofeedback studies.

The trade-off between maximizing power and controlling for all possible confounds is one of the challenges of neurofeedback study design. As explained in Button et al. (2013) (Button et al., 2013), adequately powered studies are needed to ensure reproducibility of results. However, even assuming a large effect size of $d=0.8$, a study contrasting the experimental group with a single control group requires 26 subjects per group to have an $80 \%$ chance of detecting an effect at a $\mathrm{p}<0.05$ level with a 2-tailed $t$-test. Thus, it is challenging for researchers to run fully powered neurofeedback studies even with a single control group, and running a well powered study involving more than one control group is not always feasible, particularly when the group studied is difficult to recruit (as is often the case in clinical studies involving patient populations).

Several neurofeedback studies training healthy participants have implemented a dual-control design where the experimental neurofeedback condition is compared to sham/alternative ROI feedback and a separate mental-rehearsal group. For example, neurofeedback from the insula has been found to result in increased insula activity compared to alternative ROI feedback and engaging in the identical strategy in the fMRI environment as during experimental neurofeedback (Caria et al., 2007). Furthermore, participants receiving rostrolateral prefrontal cortex neurofeedback were able to significantly increase activity in this region relative to participants receiving yoked feedback or doing mental rehearsal in the fMRI environment (McCaig et al., 2011). By using these two control conditions, these studies were able to demonstrate physiological specificity (feedback from the region is needed to regulate that region) and to rule out placebo, motivation, and non-specific effects (as the yoked or alternative ROI feedback group had the same expectations and experience of success as the experimental neurofeedback group) and to establish that simply engaging in the strategy in the absence of neurofeedback would not result in similar neurophysiological changes.

A more common approach, particularly in clinical populations, is to use one control group that receives a placebo-controlled form of feedback and is also matched for the instructed or suggested mental strategies to try during feedback (Young et al., 2017; Hamilton et al., 2016; Scheinost et al., 2013). By minimizing the number of control groups, this approach aims to maximize power while still controlling both for the assigned mental strategy and placebo/motivation effects. How precisely the mental tasks are controlled for may vary, depending on whether the mental strategies are instructed, or just recommended, on whether there are multiple strategies allowed, and on how well-defined and uniformly executed the mental strategies are.

The focus of this paper has been on control conditions for real-time fMRI neurofeedback studies. However, neurofeedback is also being done using EEG, MEG, and fNIRS. Many of the issues we discuss herein apply to neurofeedback studies in general, regardless of the functional imaging technique being implemented. Sham neurofeedback, for example, is possible across all of these techniques. Instead of an alternative region as a control condition, different frequency bands or locations (e.g., anterior vs. posterior alpha) can be used. EEG neurofeedback has been in place for decades (e.g., (Gruzelier, 2014; Micoulaud-Franchi et al., 2015) for a review). MEG and fNIRS neurofeedback are still in the early phases of development and testing, with many studies identifying as pilot studies that do not include any control condition (Sacchet et al., 2012; Foldes et al., 2015; Florin et al., 2014; Barth et al., 2016), though some have included a sham feedback control (Mihara et al., 2012; Okazaki et al., 2015; Kajal et al., 2017), or more complex designs (Marx et al., 2014).

Finally, we note that it is critical to address the issue of control between studies. fMRI protocols have been notoriously difficult to replicate and there is a 'replication crisis' in the field of neuroimaging (Open Science, 2015). The field would benefit greatly from pre-registering experiments and standardizing measures, design, statistical analysis, and reporting. A review of current best practices for neurofeedback designs can be found in (Thibault et al., 2018).

In conclusion, the best approach to designing neurofeedback studies should enable the exclusion of as many potential confounds/alternative effects as possible. In many cases, multiple control conditions will be ideal, but whether the target neurofeedback intervention should be compared with multiple control conditions in one study, or consecutively over different studies is a matter of study design that has to take power, resources, and scientific and clinical priorities into consideration. Input from clinical trial experts and statisticians will be crucial in this process. The specific goal of a particular neurofeedback study can help inform the choice of a control condition; if the goal is to determine clinical efficacy in patients, comparison to TAU or sham neurofeedback may be appropriate. If the goal, however, is to understand brain-behavior relationships in healthy individuals, bidirectional, alternative ROI, or sham may be the best choice.

\section{Declaration of interest}

MH has a patent application for fNIRS neurofeedback titled "Methods and systems for treating a subject using NIRS neurofeedback" (PCT/ US2017/036532, filed June 8, 2017). All other authors declare no conflict of interest. 


\section{Acknowledgements}

This work was supported by the Swiss National Science Foundation (BSSG10_155915, 32003B_166566), the Foundation for Research in Science and the Humanities at the University of Zurich (STWF-17-012), the Baugarten Stiftung, The BRAINTRAIN project, supported by the European Commission, under the Health Cooperation Work Programme of the 7th Framework Programme, under the Grant Agreement n ${ }^{\circ}$ 602186, and National Institutes of Health/National Institute of Mental Health under Award Number R00 MH101235-03, R01 MH100068, and R01 MH095789. The content is solely the responsibility of the authors and does not necessarily represent the official views of the funding agencies.

\section{Appendix A. Supplementary data}

Supplementary data to this article can be found online at https://doi. org/10.1016/j.neuroimage.2018.11.004.

\section{References}

Alegria, A.A., Wulff, M., Brinson, H., Barker, G.J., Norman, L.J., Brandeis, D., Stahl, D., David, A.S., Taylor, E., Giampietro, V., Rubia, K., 2017. Real-time fMRI neurofeedback in adolescents with attention deficit hyperactivity disorder. Hum. Brain Mapp. 38, 3190-3209.

Alino, M., Gadea, M., Espert, R., 2016. A critical view of neurofeedback experimental designs: sham and control as necessary conditions. Int. J. Neurol. Neurother. 3, 41.

Allison, B., Beuper, C., 2010. Could anyone use a BCI?. In: Brain-computer Interfaces. Springer, Washington, USA, pp. 35-54.

Barth, B., Strehl, U., Fallgatter, A.J., Ehlis, A.C., 2016. Near-infrared spectroscopy based neurofeedback of prefrontal cortex activity: a proof-of-concept study. Front. Hum. Neurosci. 10, 633.

Baumeister, S., Wolf, I., Holz, N., Boecker-Schlier, R., Adamo, N., Holtmann, M., Ruf, M. Banaschewski, T., Hohmann, S., Brandeis, D., 2016. Neurofeedback training effects on inhibitory brain activation in ADHD: a matter of learning? Neuroscience 378, 89-99.

Blankstein, K.R., Zimmerman, J., Egner, K., 1976. Within-subject control designs and voluntary bidirectional control of cardiac rate: methodological comparison between pre-experiment and pretrial baselines. J. Gen. Psychol. 95, 161-175.

Bray, S., Shimojo, S., O'Doherty, J.P., 2007. Direct instrumental conditioning of neural activity using functional magnetic resonance imaging-derived reward feedback. J. Neurosci. 27, 7498-7507.

Brody, S., Rau, H., Kohler, F., Schupp, H., Lutzenberger, W., Birbaumer, N., 1994. Slow cortical potential biofeedback and the startle reflex. Biofeedback Self-Regul. 19, 1-11.

Burns, T., 2009. End of the road for treatment-as-usual studies? Br. J. Psychiatry 195, 5-6.

Button, K.S., Ioannidis, J.P., Mokrysz, C., Nosek, B.A., Flint, J., Robinson, E.S.,

Munafo, M.R., 2013. Power failure: why small sample size undermines the reliability of neuroscience. Nat. Rev. Neurosci. 14, 365-376.

Buyukturkoglu, K., Roettgers, H., Sommer, J., Rana, M., Dietzsch, L., Arikan, E.B., Veit, R., Malekshahi, R., Kircher, T., Birbaumer, N., Sitaram, R., Ruiz, S., 2015. Self-regulation of anterior insula with real-time fMRI and its behavioral effects in obsessivecompulsive disorder: a feasibility study. PLoS One 10, e0135872.

Caria, A., Veit, R., Sitaram, R., Lotze, M., Weiskopf, N., Grodd, W., Birbaumer, N., 2007. Regulation of anterior insular cortex activity using real-time fMRI. Neuroimage 35, 1238-1246.

Caria, A., Sitaram, R., Veit, R., Begliomini, C., Birbaumer, N., 2010. Volitional control of anterior insula activity modulates the response to aversive stimuli. A real-time functional magnetic resonance imaging study. Biol. Psychiatry 68, 425-432.

Carlson, R.V., Boyd, K.M., Webb, D.J., 2004. The revision of the Declaration of Helsinki: past, present and future. Br. J. Clin. Pharmacol. 57, 695-713.

Chiew, M., LaConte, S.M., Graham, S.J., 2012. Investigation of fMRI neurofeedback of differential primary motor cortex activity using kinesthetic motor imagery. Neuroimage 61, 21-31.

Colgan, M., 1977. Effects of binary and proportional feedback on bidirectional control of heart rate. Psychophysiology 14, 187-191.

Cortese, A., Amano, K., Koizumi, A., Kawato, M., Lau, H., 2016. Multivoxel neurofeedback selectively modulates confidence without changing perceptual performance. Nat. Commun. 7, 13669.

Cortese, A., Amano, K., Koizumi, A., Lau, H., Kawato, M., 2017. Decoded fMRI neurofeedback can induce bidirectional confidence changes within single participants. Neuroimage 149, 323-337.

Cox, W.M., Subramanian, L., Linden, D.E., Lührs, M., McNamara, R., Playle, R., Hood, K., Watson, G., Whittaker, J.R., Sakhuja, R., Ihssen, N., 2016. Neurofeedback training for alcohol dependence versus treatment as usual: study protocol for a randomized controlled trial. Trials 17,480 .

Craig, P., Dieppe, P., Macintyre, S., Michie, S., Nazareth, I., Petticrew, M., Guidance, M.R.C., 2008. Developing and evaluating complex interventions: the new Medical Research Council guidance. BMJ 337, a1655.

deBettencourt, M.T., Cohen, J.D., Lee, R.F., Norman, K.A., Turk-Browne, N.B., 2015. Closed-loop training of attention with real-time brain imaging. Nat. Neurosci. 18, $470-475$.
deCharms, R.C., Christoff, K., Glover, G.H., Pauly, J.M., Whitfield, S., Gabrieli, J.D., 2004. Learned regulation of spatially localized brain activation using real-time fMRI Neuroimage 21, 436-443.

deCharms, R.C., Maeda, F., Glover, G.H., Ludlow, D., Pauly, J.M., Soneji, D. Gabrieli, J.D., Mackey, S.C., 2005. Control over brain activation and pain learned by using real-time functional MRI. Proc. Natl. Acad. Sci. U. S. A. 102, 18626-18631.

Florin, E., Bock, E., Baillet, S., 2014. Targeted reinforcement of neural oscillatory activity with real-time neuroimaging feedback. Neuroimage 88, 54-60.

Foldes, S.T., Weber, D.J., Collinger, J.L., 2015. MEG-based neurofeedback for hand rehabilitation. J. NeuroEng. Rehabil. 12, 85.

Furukawa, T.A., Noma, H., Caldwell, D.M., Honyashiki, M., Shinohara, K., Imai, H., Chen, P., Hunot, V., Churchill, R., 2014. Waiting list may be a nocebo condition in psychotherapy trials: a contribution from network meta-analysis. Acta Psychiatr. Scand. 130, 181-192.

Garrison, K.A., Scheinost, D., Worhunsky, P.D., Elwafi, H.M., Thornhill, TAt, Thompson, E., Saron, C., Desbordes, G., Kober, H., Hampson, M., Gray, J.R., Constable, R.T., Papademetris, X., Brewer, J.A., 2013. Real-time fMRI links subjective experience with brain activity during focused attention. Neuroimage 81, 110-118.

Gerin, M.I., Fichtenholtz, H., Roy, A., Walsh, C.J., Krystal, J.H., Southwick, S., Hampson, M., 2016. Real-time fMRI neurofeedback with war veterans with chronic PTSD: a feasibility study. Front. Psychiatr. 7, 111.

Greer, S.M., Trujillo, A.J., Glover, G.H., Knutson, B., 2014. Control of nucleus accumbens activity with neurofeedback. Neuroimage 96, 237-244.

Gruzelier, J.H., 2014. EEG-neurofeedback for optimising performance. I: a review of cognitive and affective outcome in healthy participants. Neurosci. Biobehav. Rev. 44, 124-141.

Gruzelier, J.H., Thompson, T., Redding, E., Brandt, R., Steffert, T., 2014. Application of alpha/theta neurofeedback and heart rate variability training to young contemporary dancers: state anxiety and creativity. Int. J. Psychophysiol. 93, 105-111.

Guan, M., Ma, L., Li, L., Yan, B., Zhao, L., Tong, L., Dou, S., Xia, L., Wang, M., Shi, D., 2015. Self-regulation of brain activity in patients with postherpetic neuralgia: a double-blind randomized study using real-time FMRI neurofeedback. PLoS One 10, e0123675.

Habes, I., Rushton, S., Johnston, S.J., Sokunbi, M.O., Barawi, K., Brosnan, M., Daly, T., Ihssen, N., Linden, D.E., 2016. fMRI neurofeedback of higher visual areas and perceptual biases. Neuropsychologia 85, 208-215.

Hamilton, J.P., Glover, G.H., Hsu, J.J., Johnson, R.F., Gotlib, I.H., 2011. Modulation of subgenual anterior cingulate cortex activity with real-time neurofeedback. Hum. Brain Mapp. 32, 22-31.

Hamilton, J.P., Glover, G.H., Bagarinao, E., Chang, C., Mackey, S., Sacchet, M.D., Gotlib, I.H., 2016. Effects of salience-network-node neurofeedback training on affective biases in major depressive disorder. Psychiatr. Res. 249, 91-96.

Harmelech, T., Friedman, D., Malach, R., 2015. Differential magnetic resonance neurofeedback modulations across extrinsic (visual) and intrinsic (default-mode) nodes of the human cortex. J. Neurosci. 35, 2588-2595.

Hartwell, K.J., Hanlon, C.A., Li, X., Borckardt, J.J., Canterberry, M., Prisciandaro, J.J., Moran-Santa Maria, M.M., LeMatty, T., George, M.S., Brady, K.T., 2016. Individualized real-time fMRI neurofeedback to attenuate craving in nicotinedependent smokers. J. Psychiatr. Neurosci.: J. Psychiatr. Neurosci. 41, 48-55.

Hawkinson, J.E., Ross, A.J., Parthasarathy, S., Scott, D.J., Laramee, E.A., Posecion, L.J., Rekshan, W.R., Sheau, K.E., Njaka, N.D., Bayley, P.J., deCharms, R.C., 2012. Quantification of adverse events associated with functional MRI scanning and with real-time fMRI-based training. Int. J. Behav. Med. 19, 372-381.

Hinterberger, T., Veit, R., Strehl, U., Trevorrow, T., Erb, M., Kotchoubey, B., Flor, H., Birbaumer, N., 2003. Brain areas activated in fMRI during self-regulation of slow cortical potentials (SCPs). Exp. Brain Res. 152, 113-122.

Johnson, K.A., Hartwell, K., LeMatty, T., Borckardt, J., Morgan, P.S., Govindarajan, K., Brady, K., George, M.S., 2012. Intermittent "real-time" fMRI feedback is superior to continuous presentation for a motor imagery task: a pilot study. J. Neuroimaging 22 , $58-66$.

Johnston, S., Linden, D.E., Healy, D., Goebel, R., Habes, I., Boehm, S.G., 2011 Upregulation of emotion areas through neurofeedback with a focus on positive mood. Cognit. Affect Behav. Neurosci. 11, 44-51.

Kajal, D.S., Braun, C., Mellinger, J., Sacchet, M.D., Ruiz, S., Fetz, E., Birbaumer, N., Sitaram, R., 2017. Learned control of inter-hemispheric connectivity: effects on bimanual motor performance. Hum. Brain Mapp. 38, 4353-4369.

Kluetsch, R.C., Ros, T., Theberge, J., Frewen, P.A., Calhoun, V.D., Schmahl, C., Jetly, R., Lanius, R.A., 2014. Plastic modulation of PTSD resting-state networks and subjective wellbeing by EEG neurofeedback. Acta Psychiatr. Scand. 130, 123-136.

Koizumi, A., Amano, K., Cortese, A., Shibata, K., Yoshida, W., Seymour, B., Kawato, M., Lau, H., 2016. Fear reduction without fear through reinforcement of neural activity that bypasses conscious exposure. Nat Hum Behav 1.

Kotchoubey, B., Strehl, U., Uhlmann, C., Holzapfel, S., Konig, M., Froscher, W., Blankenhorn, V., Birbaumer, N., 2001. Modification of slow cortical potentials in patients with refractory epilepsy: a controlled outcome study. Epilepsia 42, 406-416.

Kouijzer, M.E., van Schie, H.T., Gerrits, B.J., Buitelaar, J.K., de Moor, J.M., 2013. Is EEGbiofeedback an effective treatment in autism spectrum disorders? A randomized controlled trial. Appl. Psychophysiol. Biofeedback 38, 17-28.

Koush, Y., Rosa, M.J., Robineau, F., Heinen, K., S, W.R., Weiskopf, N., Vuilleumier, P., Van De Ville, D., Scharnowski, F., 2013. Connectivity-based neurofeedback: dynamic causal modeling for real-time fMRI. Neuroimage 81, 422-430.

Krause, F., Benjamins, C., Lührs, M., Eck, J., Noirhomme, Q., Rosenke, M., Brunheim, S., Sorger, B., Goebel, R., 2017. Real-time fMRI-based self-regulation of brain activation across different visual feedback presentations. Brain-Comput. interfaces 4, 87-101.

Kravitz HM, Esty ML, Katz RS, Fawcett J. Treatment of fibromyalgia syndrome using lowintensity neurofeedback with the flexyx neurotherapy system: a randomized controlled clinical trial. J. Neurother.. 2996;10:41-58. 
Lawrence, E.J., Su, L., Barker, G.J., Medford, N., Dalton, J., Williams, S.C., Birbaumer, N., Veit, R., Ranganatha, S., Bodurka, J., Brammer, M., Giampietro, V., David, A.S., 2014. Self-regulation of the anterior insula: reinforcement learning using real-time fMRI neurofeedback. Neuroimage 88, 113-124.

Lee, J.H., Kim, J., Yoo, S.S., 2012. Real-time fMRI-based neurofeedback reinforces causality of attention networks. Neurosci. Res. 72, 347-354.

Li, X., Hartwell, K.J., Borckardt, J., Prisciandaro, J.J., Saladin, M.E., Morgan, P.S., Johnson, K.A., Lematty, T., Brady, K.T., George, M.S., 2013. Volitional reduction of anterior cingulate cortex activity produces decreased cue craving in smoking cessation: a preliminary real-time fMRI study. Addict. Biol. 18, 739-748.

Li, Z., Tong, L., Wang, L., Li, Y., He, W., Guan, M., Yan, B., 2016. Self-regulating positive emotion networks by feedback of multiple emotional brain states using real-time fMRI. Exp. Brain Res. 234, 3575-3586.

Linden, D.E., Habes, I., Johnston, S.J., Linden, S., Tatineni, R., Subramanian, L., Sorger, B., Healy, D., Goebel, R., 2012. Real-time self-regulation of emotion networks in patients with depression. PLoS One 7, e38115.

MacInnes, J.J., Dickerson, K.C., Chen, N.K., Adcock, R.A., 2016. Cognitive neurostimulation: learning to volitionally sustain ventral tegmental area activation. Neuron 89, 1331-1342.

Marins, T.F., Rodrigues, E.C., Engel, A., Hoefle, S., Basilio, R., Lent, R., Moll, J., TovarMoll, F., 2015. Enhancing motor network activity using real-time functional MRI neurofeedback of left premotor cortex. Front. Behav. Neurosci. 9, 341.

Marx, A.M., Ehlis, A.C., Furdea, A., Holtmann, M., Banaschewski, T., Brandeis, D., Rothenberger, A., Gevensleben, H., Freitag, C.M., Fuchsenberger, Y., Fallgatter, A.J., Strehl, U., 2014. Near-infrared spectroscopy (NIRS) neurofeedback as a treatment for children with attention deficit hyperactivity disorder (ADHD)-a pilot study. Front. Hum. Neurosci. 8, 1038.

Maurizio, S., Liechti, M.D., Heinrich, H., Jancke, L., Steinhausen, H.C., Walitza, S., Brandeis, D., Drechsler, R., 2014. Comparing tomographic EEG neurofeedback and EMG biofeedback in children with attention-deficit/hyperactivity disorder. Biol. Psychol. 95, 31-44.

McCaig, R.G., Dixon, M., Keramatian, K., Liu, I., Christoff, K., 2011. Improved modulation of rostrolateral prefrontal cortex using real-time fMRI training and meta-cognitive awareness. Neuroimage 55, 1298-1305.

Megumi, F., Yamashita, A., Kawato, M., Imamizu, H., 2015. Functional MRI neurofeedback training on connectivity between two regions induces long-lasting changes in intrinsic functional network. Front. Hum. Neurosci. 9, 160.

Micoulaud-Franchi, J.A., McGonigal, A., Lopez, R., Daudet, C., Kotwas, I., Bartolomei, F., 2015. Electroencephalographic neurofeedback: level of evidence in mental and brain disorders and suggestions for good clinical practice. Neurophysiol. Clin. 45, 423-433.

Mihara, M., Miyai, I., Hattori, N., Hatakenaka, M., Yagura, H., Kawano, T., Okibayashi, M., Danjo, N., Ishikawa, A., Inoue, Y., Kubota, K., 2012. Neurofeedback using real-time near-infrared spectroscopy enhances motor imagery related cortical activation. PLoS One 7, e32234.

Moll, J., Weingartner, J.H., Bado, P., Basilio, R., Sato, J.R., Melo, B.R., Bramati, I.E., de Oliveira-Souza, R., Zahn, R., 2014. Voluntary enhancement of neural signatures of affiliative emotion using FMRI neurofeedback. PLoS One 9, e97343.

Moore, G.F., Audrey, S., Barker, M., Bond, L., Bonell, C., Hardeman, W., Moore, L., O'Cathain, A., Tinati, T., Wight, D., Baird, J., 2015. Process evaluation of complex interventions: medical Research Council guidance. BMJ 350, h1258.

Murphy, K., Bodurka, J., Bandettini, P.A., 2007. How long to scan? The relationship between fMRI temporal signal to noise ratio and necessary scan duration. Neuroimage 34, 565-574.

Ninaus, M., Kober, S.E., Witte, M., Koschutnig, K., Stangl, M., Neuper, C., Wood, G., 2013. Neural substrates of cognitive control under the belief of getting neurofeedback training. Front. Hum. Neurosci. 7, 914.

Oblak, E.F., Lewis-Peacock, J.A., Sulzer, J.S., 2017. Self-regulation strategy, feedback timing and hemodynamic properties modulate learning in a simulated fMRI neurofeedback environment. PLoS Comput. Biol. 13, e1005681.

Okazaki, Y.O., Horschig, J.M., Luther, L., Oostenveld, R., Murakami, I., Jensen, O., 2015 Real-time MEG neurofeedback training of posterior alpha activity modulates subsequent visual detection performance. Neuroimage 107, 323-332.

Open Science, C., 2015. PSYCHOLOGY. Estimating the reproducibility of psychological science. Science 349 , aac4716.

Paret, C., Kluetsch, R., Ruf, M., Demirakca, T., Hoesterey, S., Ende, G., Schmahl, C., 2014. Down-regulation of amygdala activation with real-time fMRI neurofeedback in a healthy female sample. Front. Behav. Neurosci. 8.

Paulus, J.K., Dahabreh, I.J., Balk, E.M., Avendano, E.E., Lau, J., Ip, S., 2014. Opportunities and challenges in using studies without a control group in comparative effectiveness reviews. Res. Synth. Methods 5, 152-161.

Persch, A.C., Page, S.J., 2013. Protocol development, treatment fidelity, adherence to treatment, and quality control. Am. J. Occup. Ther. 67, 146-153.

Potolicchio Jr., S.J., Zukerman, A.S., Chernigovskaya, N.V., 1979. Feedback control of human alpha rhythm from the central area. Biofeedback Self-Regul. 4, 211-219.

Randell, E., McNamara, R., Subramanian, L., Hood, K., Linden, D., 2018. Current practices in clinical neurofeedback with functional MRI - analysis of a survey using the TIDieR checklist. Eur. Psychiatr. (in press).

Regestein, Q.R., Pegram, G.V., Cook, B., Bradley, D., 1973. Alpha rhythm percentage maintained during 4- and 12-hour feedback periods. Psychosom. Med. 35, 215-222.
Rota, G., Sitaram, R., Veit, R., Erb, M., Weiskopf, N., Dogil, G., Birbaumer, N., 2009. Selfregulation of regional cortical activity using real-time fMRI: the right inferior frontal gyrus and linguistic processing. Hum. Brain Mapp. 30, 1605-1614.

Ruiz, S., Lee, S., Soekadar, S.R., Caria, A., Veit, R., Kircher, T., Birbaumer, N., Sitaram, R., 2013. Acquired self-control of insula cortex modulates emotion recognition and brain network connectivity in schizophrenia. Hum. Brain Mapp. 34, 200-212.

Sacchet, M.D., Mellinger, J., Sitaram, R., Braun, C., Birbaumer, N., Fetz, E., 2012. Volitional control of neuromagnetic coherence. Front. Neurosci. 6, 189.

Sarkheil, P., Zilverstand, A., Kilian-Hutten, N., Schneider, F., Goebel, R., Mathiak, K., 2015. fMRI feedback enhances emotion regulation as evidenced by a reduced amygdala response. Behav. Brain Res. 281, 326-332.

Scharnowski, F., Veit, R., Zopf, R., Studer, P., Bock, S., Diedrichsen, J., Goebel, R., Mathiak, K., Birbaumer, N., Weiskopf, N., 2015. Manipulating motor performance and memory through real-time fMRI neurofeedback. Biol. Psychol. 108, 85-97.

Scheinost, D., Stoica, T., Saksa, J., Papademetris, X., Constable, R.T., Pittenger, C., Hampson, M., 2013. Orbitofrontal cortex neurofeedback produces lasting changes in contamination anxiety and resting-state connectivity. Transl. Psychiatry 3 e250.

Schoenberg, P.L., David, A.S., 2014. Biofeedback for psychiatric disorders: a systematic review. Appl. Psychophysiol. Biofeedback 39, 109-135.

Sherwood, M.S., Kane, J.H., Weisend, M.P., Parker, J.G., 2016. Enhanced control of dorsolateral prefrontal cortex neurophysiology with real-time functional magnetic resonance imaging (rt-fMRI) neurofeedback training and working memory practice. Neuroimage 124, 214-223.

Sherwood, M.S., Weisend, M.P., Kane, J.H., Parker, J.G., 2016. Combining real-time fMRI neurofeedback training of the DLPFC with N-back practice results in neuroplastic effects confined to the neurofeedback target region. Front. Behav. Neurosci. 10, 138.

Shibata, K., Watanabe, T., Sasaki, Y., Kawato, M., 2011. Perceptual learning incepted by decoded fMRI neurofeedback without stimulus presentation. Science 334, 1413-1415.

Shibata, K., Watanabe, T., Kawato, M., Sasaki, Y., 2016. Differential activation patterns in the same brain region led to opposite emotional states. PLoS Biol. 14, e1002546.

Sitaram, R., Caria, A., Veit, R., Gaber, T., Ruiz, S., Birbaumer, N., 2014. Volitional control of the anterior insula in criminal psychopaths using real-time fMRI neurofeedback: a pilot study. Front. Behav. Neurosci. 8, 344.

Sorger, B., Kamp, T., Weiskopf, N., Peters, J.C., Goebel, R., 2016. When the brain takes 'BOLD' steps: real-time fMRI neurofeedback can further enhance the ability to gradually self-regulate regional brain activation. Neuroscience 378, 71-88.

Spilker, B., 1991. Guide to Clinical Trials. Raven Press, New York.

Subramanian, L., Hindle, J.V., Johnston, S., Roberts, M.V., Husain, M., Goebel, R., Linden, D., 2011. Real-time functional magnetic resonance imaging neurofeedback for treatment of Parkinson's disease. J. Neurosci. 31, 16309-16317.

Sulzer, J., Sitaram, R., Blefari, M.L., Kollias, S., Birbaumer, N., Stephan, K.E., Luft, A., Gassert, R., 2013. Neurofeedback-mediated self-regulation of the dopaminergic midbrain. Neuroimage 83, 817-825.

Sulzer, J., Haller, S., Scharnowski, F., Weiskopf, N., Birbaumer, N., Blefari, M.L., Bruehl, A.B., Cohen, L.G., DeCharms, R.C., Gassert, R., Goebel, R., Herwig, U., LaConte, S., Linden, D., Luft, A., Seifritz, E., Sitaram, R., 2013. Real-time fMRI neurofeedback: progress and challenges. Neuroimage 76, 386-399.

Teuber, H.L., 1955. Physiological psychology. Annu. Rev. Psychol. 6, 267-296.

Thibault, R.T., Lifshitz, M., Raz, A., 2016. The self-regulating brain and neurofeedback: experimental science and clinical promise. Cortex 74, 247-261.

Thibault, R.T., MacPherson, A., Lifshitz, M., Roth, R.R., Raz, A., 2018. Neurofeedback with fMRI: a critical systematic review. Neuroimage 172, 786-807.

Watanabe, T., Sasaki, Y., Shibata, K., Kawato, M., 2017. Advances in fMRI real-time neurofeedback. Trends Cognit. Sci. 21, 997-1010.

Watts, S.E., Turnell, A., Kladnitski, N., Newby, J.M., Andrews, G., 2015. Treatment-asusual (TAU) is anything but usual: a meta-analysis of CBT versus TAU for anxiety and depression. J. Affect. Disord. 175, 152-167.

Witte, M., Kober, S.E., Ninaus, M., Neuper, C., Wood, G., 2013. Control beliefs can predict the ability to up-regulate sensorimotor rhythm during neurofeedback training. Front. Hum. Neurosci. 7, 478.

Yoo, S.S., O'Leary, H.M., Fairneny, T., Chen, N.K., Panych, L.P., Park, H., Jolesz, F.A., 2006. Increasing cortical activity in auditory areas through neurofeedback functional magnetic resonance imaging. Neuroreport 17, 1273-1278.

Young, K.D., Siegle, G.J., Zotev, V., Phillips, R., Misaki, M., Yuan, H., Drevets, W.C. Bodurka, J., 2017. Randomized clinical trial of real-time fMRI amygdala neurofeedback for major depressive disorder: effects on symptoms and autobiographical memory recall. Am. J. Psychiatry 174, 748-755.

Zhang, G., Zhang, H., Li, X., Zhao, X., Yao, L., Long, Z., 2013. Functional alteration of the DMN by learned regulation of the PCC using real-time fMRI. IEEE Trans. Neural Syst. Rehabil. Eng. 21, 595-606.

Zilverstand, A., Sorger, B., Sarkheil, P., Goebel, R., 2015. fMRI neurofeedback facilitates anxiety regulation in females with spider phobia. Front. Behav. Neurosci. 9, 148.

Zilverstand, A., Sorger, B., Slaats-Willemse, D., Kan, C.C., Goebel, R., Buitelaar, J.K., 2017. fMRI neurofeedback training for increasing anterior cingulate cortex activation in adult attention deficit hyperactivity disorder. An exploratory randomized, singleblinded study. PLoS One 12, e0170795. 